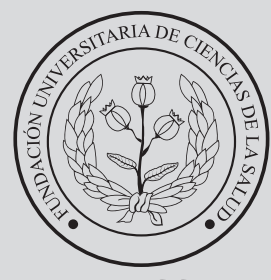

FUCS
Repe por

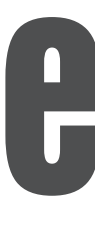

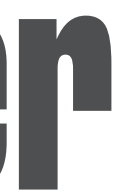
to

de

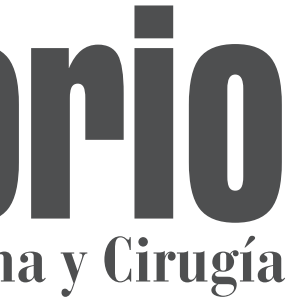

Vol. 29
No3 2020

ISSN: 0121-7372 • ISSN electrónico: 2462-991X

Artículo de investigación

\title{
Factores que impactan en la mortalidad de los pacientes con trauma que ingresan al servicio de urgencias
}

\begin{abstract}
Miguel Ángel Saavedra $\mathbf{M D}^{\mathrm{a}}$ Eliana Santacruz Castillo MD $^{\mathrm{b}}$ Katherine Rueda MD Manuel Fernando Paternina $\mathbf{M D}^{\mathrm{b}}$ Jessica Mendoza Alvaran MD $^{\mathrm{b}}$
\end{abstract}

\section{Factors associated with mortality in trauma patients admitted to the emergency service}

${ }^{a}$ Servicio de Medicina de Urgencias Hospital de San José, Cirugía General, Especialista en Cirugía Vascular, Fundación Universitaria de Ciencias de la Salud. Bogotá DC, Colombia.

${ }^{b}$ Medicina de Urgencias Fundación Universitaria de Ciencias de la Salud, Bogotá DC, Colombia.

\section{R E S U M E N}

Introducción: el trauma se ubica dentro de las primeras causas de mortalidad a nivel mundial; tener un mayor conocimiento permite crear estrategias con el fin de disminuir la mortalidad y las complicaciones, mejorando la calidad de atención y la respuesta oportuna en los servicios de urgencias. Objetivo: determinar los factores que impactan en la mortalidad de los pacientes con diagnóstico de trauma que ingresaron al servicio de urgencias de los hospitales de San José e Infantil Universitario de San José entre 2012 y 2018. Métodos: estudio de casos y controles donde fueron evaluados 462 pacientes, 47 fueron casos y 415 controles, recolectados a través de revisión de historias clínicas y analizados a partir de modelo de regresión logística. Resultados: se analizaron 462 pacientes con diagnóstico de trauma con una mediana de edad de 33 (RIQ 25-50), mayor proporción del sexo masculino $70.78 \%(n=327)$, se observó la asociación de mortalidad con las variables: edad OR 1.035 (IC 95\% 1.002- 10.70), tensión arterial sistólica OR 0.96 (IC 95\% 0.94-0.99) y Glasgow OR 0.55 (IC 95\% 0.45-0.67). El trauma craneoencefálico $(74.47 \%)$ y el accidente de tránsito $(44.68 \%)$ fueron los prevalentes. Conclusiones: este estudio determinó que existe asociación entre la edad, escala de Glasgow, tensión arterial sistólica y el desenlace de mortalidad en pacientes traumatizados.

Palabras clave: mortalidad, variables fisiológicas frecuencia cardiaca, trauma.

(C) 2020 Fundación Universitaria de Ciencias de la Salud - FUCS. Este es un artículo Open Access bajo la licencia CC BY-NC-ND (http://creativecommons.org/licenses/by-nc-nd/4.0/).

INFORMACIÓN DEL ARTÍCULO

Historia del artículo:

Fecha recibido: mayo 24 de 2019 Fecha aceptado: febrero 28 de 2020
Autor para correspondencia. Dr. Miguel Ángel Saavedra miguelangelsa@hotmail.com
DOI

10.31260/RepertMedCir.01217372.913 
Introduction: traumatic injury is one of the leading causes of death worldwide. Gaining knowledge on this topic is the basis for developing strategies to decrease mortality and complications, improving the quality of care and providing a timely response at the emergency services. Objective: to determine the factors associated with mortality in trauma patients admitted to the emergency service of San José and Infantil Universitario de San José hospitals between 2012 and 2018. Methods: a case and control study including 462 patients, 47 cases and 415 controls, collected in a clinical record review and analyzed using a logistic regression model. Results: we analyzed 462 trauma patients. Median age was 33 (IQR 25-50), and 70.78\% $(\mathrm{n}=327)$ were males. We observed the relationship between age OR 1.035 (CI 95\% 1.002- 10.70), systolic blood pressure OR 0.96 (CI 95\% 0.94-0.99) and Glasgow coma score OR 0.55 (CI 95\% 0.45-0.67) with mortality. The most frequent cause was cranioencephalic trauma $(74.47 \%)$ and traffic accidents $(44.68 \%)$. Conclusions: this study determined there is an association between age, Glasgow coma score and systolic blood pressure with mortality as outcome in trauma patients.

Key words: mortality, physiologic variables, cardiac rate, trauma

(C) 2020 Fundación Universitaria de Ciencias de la Salud - FUCS This is an open access article under the CC BY-NC-ND license (http://creativecommons.org/licenses/by-nc-nd/4.0/).

\section{INTRODUCCIÓN}

A nivel mundial el trauma constituye un problema de salud pública, siendo la primera causa de mortalidad en la población entre los 15 y 45 años de edad, lo cual conlleva a que siga siendo objeto de estudio en diferentes áreas de la práctica clínica. Es evidente que el diagnosticó y el enfoque terapéutico tempranos, impactan en el desenlace. La detección oportuna de factores implicados en la severidad y la eficacia de un adecuado sistema de triage en el servicio de urgencias, permite una mejor intervención en los pacientes traumatizados, mejorando los resultados en cuanto al manejo, pronóstico y secuelas. ${ }^{1}$

De acuerdo con las estadísticas de la Organización Mundial de la Salud (OMS), la tasa de mortalidad asociada con trauma alrededor del mundo en 2011 fue 78 muertes/100.000 habitantes, reportándose en la literatura en orden decreciente accidentes de tránsito, violencia y caídas. ${ }^{2}$ Bajo la evidencia presentada y basados en la mayor cantidad de traumas, se demuestra que los torácicos causados por accidentes de tránsito representan $70 \%$ de aumento en la morbimortalidad, $25 \%$ son debidos a traumas cerrados como caída de altura y $5 \%$ restante son por lesiones penetrantes, ya sea por armas cortopunzantes o de fuego. ${ }^{2}$

Según el reporte del Ministerio de Salud y Protección Social en los datos de mortalidad expuestos para 2016 se identificó que "las causas externas se ubican en el cuarto lugar de frecuencia, entre 2005 y 2014, las agresiones (homicidios) provocaron 52.24\% (169.813) del total de muertes, para el último año las tasas ajustadas se redujeron $40 \%$, con respecto a 2005, disminuyendo a una tasa de 17,41 muertes por cada 100.000 habitantes; para 2014. ${ }^{3}$ Los accidentes de tránsito, generaron $19,57 \%(63,620)$ de las muertes, y se ubicaron en segundo lugar de frecuencia teniendo prevalencia especialmente en la población de mujeres. En tercer lugar, las lesiones autoinfligidas (suicidios) disminuyeron 12\%". Partiendo de dicho reporte se logra identificar que para 2020 las cifras de mortalidad aumentarán hasta en $20 \%$. $^{3}$

Con lo anterior y en congruencia con lo expuesto por el Dr. Gosselin RA en 2009, se define que los traumatismos son una epidemia desatendida en los países en desarrollo, causando más de cinco millones de muertes al año. A pesar de la recopilación de la información no hay datos concretos sobre el número de personas que sobreviven y aún más, se desconoce el número con algún tipo de discapacidad permanente asociada con el traumatismo. ${ }^{4}$ Otro factor que podría resaltarse son los costos que implican, teniendo en cuenta los gastos de atención clínica, rehabilitación, incapacidades y pensión de invalidez o muerte. ${ }^{4}$

En búsqueda de caracterización sobre la mortalidad en trauma, Trunkey en 1983 identificó y propuso una distribución trimodal, donde sugiere que 50\% de las muertes ocurren inmediatamente después del accidente causado por trauma craneoencefálico o de tórax (grandes vasos), catalogado como el primer pico (10 primeros minutos), por otro lado $30 \%$ fallecen en las primeras horas por acidosis, hipoxia e hipovolemia considerado el segundo pico, y $20 \%$ hasta días o semanas después considerado el tercer pico debido a sepsis y falla multiorgánica. Por lo tanto una alta proporción de muertes ocurren durante las primeras horas del trauma y pueden prevenirse con intervención médica temprana. ${ }^{5}$

Con el pasar de los años y de la estructuración de los servicios de urgencias, se ha buscado promover el entrenamiento de los profesionales para la atención del paciente traumatizado, es por ello que el Comité de Trauma del Colegio Americano de Cirujanos fue autorizado desde 1995 a impartir el curso ATLS (Advanced Trauma Life 
Support), con el objetivo de entrenar a todos los médicos asistenciales del servicio de urgencias en el manejo rápido y eficiente del paciente con trauma múltiple durante la "hora dorada". ${ }^{6}$ De esta manera se han creado estrategias de abordaje y valoración del paciente traumatizado, enfatizando en elementos vitales como el ABCDE en revisión primaria y secundaria; dichos programas fueron creados por la World Federation of Societies of Anaesthesiologists y avalados por la Organización Mundial de la Salud (OMS) con el objetivo fundamental de reducir la morbilidad y la mortalidad. ${ }^{5}$

El fin de esta investigación fue determinar si las variables fisiológicas, sociodemográficas, la escala de Glasgow, los mecanismos de trauma y la zona anatómica tuvieron impacto sobre la mortalidad de los pacientes que ingresaron al servicio de urgencias de los hospitales de San José e Infantil Universitario de San José, entre 2012 y 2018 con diagnóstico de trauma.

\section{MATERIALES Y MÉTODOS}

\section{Diseño del estudio}

Se llevó a cabo un estudio de casos y controles los primeros se definieron como pacientes que ingresaron al servicio de urgencias con diagnóstico de traumatismo y que su condición al egreso fuese muerte, y como controles aquellos que ingresaron por traumatismos y que su condición al egreso fuese vivo, en el periodo comprendido entre 2012 y 2018 en los hospitales de San José e Infantil Universitario de San José.

\section{Criterios de elegibilidad}

Los criterios de inclusión fueron los definidos en el diseño del estudio. Los de exclusión fueron los pacientes con condiciones clínicas avanzadas como insuficiencia cardiaca, cardiopatía isquémica, fallas renal o hepática que pudieran acelerar el proceso de muerte. También se excluyeron a quienes no se pudo realizar seguimiento en la historia clínica, debido a que el egreso no fue por alta médica (remisión y salida voluntaria). Para el análisis se midieron variables demográficas y clínicas como sexo, edad, mecanismo del trauma, zona anatómica, Glasgow, frecuencias cardiaca y respiratoria, así como tensión arterial sistólica, con el fin de evaluar la asociación de dichos factores con el desenlace de muerte en los pacientes con diagnóstico de trauma que ingresaron al servicio de urgencias en este período.

\section{Participantes}

Se tomaron 462 pacientes del estudio "Características operativas de la triada letal en pacientes que ingresaron al servicio de urgencias con diagnóstico de trauma mayor para predecir mortalidad"7, de los cuales 415 fueron los controles y 47 fueron los casos. Se realizó una muestra consecutiva de tal forma que los datos fueron recolectados durante un periodo específico entre los años 2012 a 2018.

\section{Recolección de datos y análisis estadístico}

Los datos se obtuvieron directamente de las historias clínicas, construyendo una base de datos en excel 2013, la cual se exportó al software estadístico stata13, teniendo en cuenta que para las variables de zona anatómica se tomaron las de mayor impacto, descartando lesiones secundarias asociadas. Se realizó un análisis descriptivo de las variables demográficas y clínicas usando mediana y rango intercuartílico para las variables cuantitativas, y frecuencias absolutas y relativas para las cualitativas. Se realizaron análisis bivariados para identificar posibles factores de riesgo de mortalidad. En las variables continuas se usó la prueba no paramétrica de Mann-Whitney, por no cumplirse el supuesto de normalidad el cual fue evaluado mediante la prueba de ShapiroWilk. Para las variables categóricas se construyeron tablas de contingencia y se realizaron pruebas de independencia usando la prueba chi cuadrado. Se calcularon medidas de efecto (OR) ajustadas mediante un modelo de regresión logística multivariado. Para el ajuste se usaron las variables con resultados significativos en el análisis bivariado $(\mathrm{p}<0.2)$. Para las variables mecanismo de trauma y zona anatómica se tomaron como grupos de referencia las categorías con menor número de observaciones, "herida por arma de fuego" y "extremidades", respectivamente.

\section{CONSIDERACIONES ÉTICAS}

En este estudio se tuvo en cuenta la norma científica, técnica y administrativa para la investigación en salud en seres humanos contenidas en la resolución 8430 de 1993 del Ministerio de Salud. Según el artículo 11 se considera que esta investigación puede clasificarse como "SIN RIESGO". ${ }^{8}$ Fue presentada al comité de ética de la Fundación Universitaria de Ciencias de la Salud, quien validó información y aprobó la ejecución del protocolo de investigación por parte del grupo investigador.

\section{RESULTADOS}

Durante el periodo de la investigación fueron evaluados 462 pacientes, de los cuales $\mathrm{n}=415$ correspondieron a controles y $\mathrm{n}=47$ a casos. El $68.92 \%$ de los controles $(\mathrm{n}=$ 286) y $87.23 \%$ de los casos $(n=41)$ correspondieron al sexo masculino, con una mediana de edad para los casos de 49 (RIQ 31-61), y los controles 33 (RIQ 25 -47). Con respecto a la escala de Glasgow la mediana de los casos fue 7 (RIQ 3-11) y controles 15 (RIQ 15 - 15). Para la frecuencia cardiaca la mediana de los casos fue de 84 (RIQ 70 - 98) y controles 83 (RIQ 78 -90), y la frecuencia respiratoria obtuvo una mediana de 18 (RIQ 16 - 20) para los casos y de 18 (RIQ 16- 20) para los controles. En relación con la tensión arterial sistólica, en los casos la mediana fue 115 (RIQ 93 - 130) y controles 120 (RIQ $110-128$ ) (tabla 1). 
Con respecto a la zona anatómica el sitio lesionado más frecuente en los casos fue "cabeza" representado por $74.47 \%$ $(\mathrm{n}=35)$ y para los controles $47.23 \%(\mathrm{n}=196)$. El mecanismo más común para los casos fue "accidente de tránsito" $48.68 \%(n=21)$ y para controles trauma "contundente" con $37.35 \%(n=155)($ tabla 1$)$.

Tabla 1. Características generales

\begin{tabular}{|c|c|c|c|}
\hline Variables & Casos $(n=47)$ & $\begin{array}{l}\text { Controles } \\
(n=415)\end{array}$ & $\mathbf{P}$ \\
\hline \multicolumn{4}{|l|}{ Sexo } \\
\hline Femenino & $6(12.77)$ & $129(31.08)$ & \multirow[t]{2}{*}{0.009} \\
\hline Masculino & $41(87.23)$ & $286(68.92)$ & \\
\hline Edad (mediana/RIC) & $49(31-61)$ & $33(25-47)$ & 0.0003 \\
\hline TAS, mediana (RIC) & $115(93-130)$ & $120(110-128)$ & 0.102 \\
\hline $\begin{array}{l}\text { Frecuencia cardiaca, } \\
\text { mediana, (RIC) }\end{array}$ & $84(70-98)$ & $83(78-90)$ & 0.787 \\
\hline $\begin{array}{l}\text { Frecuencia respiratoria, } \\
\text { mediana, (RIC) }\end{array}$ & $18(16-20)$ & $18(16-20)$ & 0.399 \\
\hline Glasgow, mediana, (RIC) & $7(3-11)$ & $15(15-15)$ & 0.0000 \\
\hline \multicolumn{4}{|l|}{ Zona anatómica n (\%) } \\
\hline Cabeza & $35(74.47)$ & $196(47.23)$ & \multirow{4}{*}{0.000} \\
\hline Tórax & $4(8.51)$ & $114(27.47)$ & \\
\hline Abdomen & $3(6.38)$ & $103(24.82)$ & \\
\hline Extremidades & $5(10.64)$ & $2(0.48)$ & \\
\hline \multicolumn{4}{|l|}{ Mecanismo trauma n (\%) } \\
\hline Accidente de tránsito & $21(44.68)$ & $61(14.70)$ & \multirow{5}{*}{0.000} \\
\hline Caída altura & $14(29.79)$ & $58(13.98)$ & \\
\hline Arma cortopunzante & $4(8.51)$ & $134(32.29)$ & \\
\hline Contundente & $2(4.26)$ & $155(37.35)$ & \\
\hline Herida arma fuego & $6(12.77)$ & $7(1.69)$ & \\
\hline
\end{tabular}

Tabla 2. Distribución anatómica con relación al mecanismo de trauma

\begin{tabular}{|c|cccccc|}
\hline $\begin{array}{c}\text { Zona } \\
\text { anatómica }\end{array}$ & $\begin{array}{c}\text { Extremidades } \\
\mathbf{n}(\%)\end{array}$ & $\begin{array}{c}\text { Tórax } \\
\mathbf{n}(\%)\end{array}$ & $\begin{array}{c}\text { Abdomen } \\
\mathbf{n}(\%)\end{array}$ & $\begin{array}{c}\text { Cabeza } \\
\mathbf{n}(\%)\end{array}$ & $\begin{array}{c}\text { Total } \\
\mathbf{n}(\%)\end{array}$ \\
\hline $\begin{array}{c}\text { Arma de } \\
\text { fuego }\end{array}$ & $1(7.69)$ & $2(15.38)$ & $4(30.77)$ & $6(46.15)$ & $13(100)$ \\
\hline $\begin{array}{c}\text { Caída de } \\
\text { altura }\end{array}$ & $1(1.39)$ & $1(1.39)$ & $32(44.44)$ & $38(52.78)$ & $72(100)$ \\
\hline $\begin{array}{c}\text { Arma } \\
\text { Gortopunzante }\end{array}$ & $0(0.0)$ & $60(43.48$ & $10(7.25)$ & $68(49.28)$ & $138(100)$ \\
\hline \begin{tabular}{c} 
Contundente \\
\hline Accidente de \\
tránsito
\end{tabular} & $0(0.0)$ & $54(34.39)$ & $11(7.01)$ & $92(58.60)$ & $157(100)$ \\
\hline & $5(6.10)$ & $1(1.22)$ & $49(50.76)$ & $27(32.93)$ & $82(100)$ \\
\hline Total & $7(1.52)$ & $118(25.54)$ & $106(22.94)$ & $231(50)$ & $462(100)$ \\
\hline
\end{tabular}

En cuanto al mecanismo del trauma y su relación con la zona anatómica, de forma global se pudo evidenciar que el trauma contundente (n: 157, 33.9\%) fue el mecanismo que se presentó con mayor frecuencia y la zona anatómica más afectada fue cabeza (n: 231, 50\%), seguido de herida por arma cortopunzante (n: 138, 29.8\%) y tórax (n: 118, $25.54 \%$ ) como la segunda zona anatómica más afectada (tabla 2).

Respecto a los resultados de análisis de regresión logística las variables con resultado significativo en el análisis bivariado $(p<0.2)$ fueron sexo, edad, tensión arterial sistólica, Glasgow, mecanismo de trauma y zona anatómica; estas variables fueron incluidas en el modelo multivariado en el cual no se incluyeron frecuencias cardiaca y respiratoria, debido a que en el análisis no tuvieron significancia estadística.

Se observó un OR 1.03 (IC 95\% 1.002-1.070) para la edad, lo que significa que por cada año de edad que aumente, incrementa el riesgo 1.035 veces la mortalidad. En la escala de Glasgow se evidenció un OR 0.55 (IC 95\% 0.45-0.67), lo que quiere decir que por cada punto que incrementa en la escala disminuye 0.5 veces el riesgo de mortalidad secundario a trauma. En relación con la tensión arterial se documentó un OR 0.96 (IC 95\% 0.94-0.99) con lo que se concluye que por cada $1 \mathrm{~mm} \mathrm{Hg}$ que aumente la tensión arterial sistólica disminuye 0.96 veces el riesgo de muerte.

Respecto al mecanismo de trauma y mortalidad tomando como comparador herida por arma de fuego, se estableció que el trauma por caída de altura, arma cortopunzante y trauma contundente presentaron menos riesgo de mortalidad, con significancia estadística. En cuanto a la zona anatómica y mortalidad tomando como comparador extremidades, se concluyó que "abdomen" tiene menor riesgo de mortalidad; ya que "tórax" y "cabeza" no tuvieron diferencias estadísticamente significativas con el grupo comparador (tabla 3).

Tabla 3. Modelo de regresión logística

\begin{tabular}{|l|c|c|c|}
\hline \multicolumn{1}{|c|}{ Variables } & Odds Ratio & IC $95 \%$ & $P$ \\
\hline Sexo & 2.46 & $0.58-10.43$ & 0.22 \\
TAS & 1.035 & $1.002-1.070$ & 0.035 \\
Glasgow & 0.96 & $0.94-0.99$ & 0.019 \\
Zona anatómica & 0.55 & $0.45-0.67$ & 0.000 \\
$\quad$ Cabeza & & & \\
Tórax & 0.42 & $0,044-3.92$ & 0.44 \\
$\quad$ Abdomen & 0.11 & $0.004-3.16$ & 0.20 \\
Mecanismo trauma & 0.033 & $0.003-0.37$ & 0.006 \\
$\quad$ Accidente de tránsito & 0.33 & $0.048-2.31$ & 0.26 \\
Caída altura & 0.06 & $0.006-0.56$ & 0.014 \\
Arma cortopunzante & 0.011 & $0.0008-0.14$ & 0.001 \\
Contundente & 0.0035 & $0.048-2.31$ & 0.26 \\
\hline
\end{tabular}

\section{DISCUSIÓN}

En el presente estudio se buscó establecer qué factores impactaron en la mortalidad de pacientes que ingresaron al servicio de urgencias por trauma, se realizó un estudio de casos y controles a partir de base de datos obtenida 
de las historias clínicas de pacientes registrados en dos centros de atención en la ciudad de Bogotá durante 6 años, demostrando que el sexo, edad, tensión arterial sistólica, escala Glasgow, mecanismo de trauma y zona anatómica fueron las variables que se asociaron con mayor riesgo de mortalidad y se estableció que en concordancia con la literatura mundial, el sexo masculino fue la población más afectada con una proporción hombre: mujer $2.5: 1 .^{9}$ Además se observó una asociación entre edad y mortalidad ya que a medida que envejece la población tienen mayores comorbilidades acumulables y que asociado al trauma tendrá un mayor riesgo de mortalidad; como lo referido en el estudio realizado por Alberdi y col. donde concluyen que "Los pacientes mayores de 65 años son una cohorte creciente. Para niveles lesionales similares tienen el doble de mortalidad que los jóvenes, debido a su elevada comorbilidad y los tratamientos asociados". ${ }^{10}$

La tensión arterial sistólica estableció una relación de riesgo con la mortalidad dado que conforme incrementa esta, se redujo el riesgo de muerte con significancia estadística; hallazgos que son comparables con el estudio de Prashant Bhandarkar y col. donde se determinó una tasa de mortalidad de $24 \%$, siendo mayor en los pacientes que cursaban al ingreso con hipotensión y bradicardia. ${ }^{11}$

Así mismo se registró que el tipo de trauma más prevalente fue el craneoencefálico con $58.6 \%$ del total de la muestra, siendo el contundente el mecanismo más común. Cuando se estableció relación de riesgo de escala de Glasgow con mortalidad, se evidenció que a medida que incrementa el puntaje se redujo la mortalidad. Citando el artículo de Alberdi F, García I, en el que refiere que el trauma grave es la sexta causa de muerte y la quinta de discapacidad en el mundo, siendo el craneal el más incidente, con mayor morbimortalidad y secuelas más graves y duraderas. ${ }^{10}$ En el artículo de Luna y col. ${ }^{12}$ concluyeron que $24 \%$ de la población correspondió a trauma craneoencefálico, datos que se correlacionan con los de nuestra población.

Los resultados pudieron verse afectados debido a que los traumas en extremidades se asociaron con lesiones vasculares ${ }^{13}$; como en el caso de un paciente que ingresó al servicio de urgencias por accidente de tránsito con fractura abierta de tibia y peroné derechos, y al ser llevado a procedimiento quirúrgico evidenciaron lesión vascular de $50 \%$ de la arteria tibial posterior y peronea derecha, llevando a choque hipovolémico refractario al tratamiento y muerte.

En el presente estudio se declaran varias limitaciones, entre ellas el registro de la historia clínica incompleta dado a que hubo información importante que no se consignó como distancia de la caída y si hubo más de un sistema comprometido, ya que solo se tuvo en cuenta la zona de mayor impacto. Otra limitación está condicionada por el poco número de eventos dentro de la muestra recolectada. Además, no hubo definición clara del mecanismo de trauma, generando heterogeneidad al momento de consignar éste en la historia clínica (estableciendo diferencia entre trauma contundente secundario a accidente de tránsito y caída de altura).

Para finalizar, la mortalidad por trauma representa una prioridad en salud pública, ya que supone para la sociedad elevados costos, teniendo en cuenta que el grupo etáreo más afectado es la población económicamente activa, generando impacto en años de vida perdidos. ${ }^{9}$

\section{CONCLUSIONES}

Este estudio determinó que existe asociación entre la edad, escala de Glasgow, tensión arterial sistólica y el desenlace de mortalidad en pacientes traumatizados. El trauma craneoencefálico y el accidente de tránsito fueron los más prevalentes en cuanto a zona anatómica y mecanismo de trauma respectivamente.

\section{CONFLICTO DE INTERÉS}

Los autores declaran no tener conflicto de intereses.

\section{FINANCIACIÓN DEL PROYECTO}

No se recibió financiación para la realización del proyecto.

\section{REFERENCIAS}

1. Cortes M C, Acuña R L, Alvarez M F, Alvarez L N. Manejo inicial del politraumatizado. Rev Hosp Clin Univ Chile. 2013;25:206-16. doi: 10.5005/jp-journals-10030-1190

2. Monteverde E, Bosque L, Lartigue B, Macia E, Barbaro C, Ortiz C, et al. Evaluacién de la Nueva Definición de Politrauma en una Cohorte de Pacientes de 10 Hospitales Argentinos. Panamerican Journal of Trauma, Critical Care \& Emergency Surgery. 2017;6(3):182-9.

3. Ministerio de Salud y Protección Social. Dirección de Epidemiología y demografía. Análisis de situación de salud (ASIS): Colombia, 2017. In: Moreno Segura CM, editor. Colombia: Imprenta Nacional de Colombia; 2018. p. 149.

4. Gosselin R, Spiegel D, Coughlin R, Lewis Z. Los traumatismos: el problema sanitario desatendido en los países en desarrollo. Boletín de la Organización Mundial de la Salud. 2009;87:246. doi: 10.2471/BLT.08.052290

5. Rivera-Flores J. El paciente traumatizado ¿Quién debe tratarlo? Rev Mex Anestesiol. 2012;35(1):5-7.

6. Graff I, Ghamari S, Schacher S, Glien P, Fimmers R, Baehner T, et al. Improvement of polytrauma management $₫$ quality inspection of a newly introduced course concept. J Eval Clin Pract. 2017;23(6):1381-6. doi: https://doi.org/10.1111/jep.12802 
7. Cerón Alate LY, Méndez Hernández J, Candela Rada J. Consideraciones sobre la tríada letal para predecir mortalidad en pacientes con trauma mayor. Repert Med Cir. 2019. doi: https:// orcid.org/10.31260/RepertMedCir.01217273.665

8. Resolución 8430: Por la cual se establecen las normas científicas, técnicas y administrativas para la investigación en salud., (1993).

9. Berroeta AF, Odriozola AF, Alzugaray TG, Fernández AI, Jiménez RE, Morán TS. Estudio epidemiológico autópsico de 784 fallecimientos por traumatismo. Proyecto POLIGUTANIA. . Med Intensiva. 2002;26(10):491-500.

10. Alberdi F, García I, Atutxa L, Zabarte M. Epidemiologia del trauma grave. Med Intensiva. 2014;38(9):580-8. doi: 10.1016/j. medin.2014.06.012
11. Bhandarkar P, Munivenkatappa A, Roy N, Kumar V, Samudrala V, Kamble J, et al. On-admission blood pressure and pulse rate in trauma patients and their correlation with mortality: Cushing\&\#39;s phenomenon revisited. Int J Crit Illn Inj Sci. 2017;7(1):14-7.

12. Luna D, Izquierdo $M$, Perez G, Rodriguez C. Incidencia de traumas por accidentes de tránsito que ingresan al servicio de urgencias del Hospital Universitario Erasmo Meoz, La Clínica Santa Ana Sa, e IPS UNIPAMPLONA. Rev Inbiom. 2017;4(1):34-43.

13. García Romero M, Patiño Mesa DA. Trauma vascular de extremidades. Urgentia R Int Med Emergencias. 2015;1(1):60-4. 